\title{
La métrologie en chimie Quelques principes et beaucoup d'incertitudes
}

\author{
J. Vialle ${ }^{1}$, P. Linet $^{1}$ et E.A. Maier ${ }^{2}$ \\ ${ }^{I}$ Service Central d'Analyse, CNRS, BP. 22, 69390 Vernaison, France \\ ${ }_{2}^{2}$ Programme Normes, Mesures et Essais, Commission Européenne, Rue de la Loi 200, B-1049 Bruxelles, Belgique
}

\begin{abstract}
The increasing need for reliable analytical measurements in chemistry is a consequence of the impact of results on socio-economic or legal decisions. Lack of reliability of analytical results, partly originates from the absence of a structured metrology system in chemistry. Ideally, such a metrology system should be able to allow to generate results traceable to the International System of units and to evaluate reliably the uncertainty. Most chemical measurements request long and complex procedures leading to physical or/and chemical treatments of samples forbidding a direct link to the kilogram or the mole. Only few analytical methods can be proposed as "primary methods" linking results directly to the fundamental units and often they cannot be used on real samples. To perform their task analytical chemists use secondary methods, validated and calibrated with reference materials, certified if possible. The way used to obtain secondary traceable methods, to develop and use reference materials, and to evaluate the uncertainty of measurement results are described and discussed.
\end{abstract}

\section{Introduction}

\section{L'actualité de l'analyse chimique}

Il est devenu une évidence que dans la plupart des domaines de l'activité socio-économique, il est fait de plus en plus appel à l'analyse chimique. Partout dans le monde, le résultat analytique est la base sur laquelle sont établies et vérifiées les hypothèses scientifiques, élaborées et contrôlées les productions industrielles, discutés des accords commerciaux, évaluées les pollutions, établis des diagnostics et des traitements médicaux, fixées des normes, et sans laquelle ne peuvent se prendre certaines décisions juridiques et politiques. On demande désormais aux analystes de fournir des éléments de réponse à des problèmes de société.

Avec l'ouverture des frontières, l'internationalisation de l'activité dans tous ces domaines ne fait que se développer. Elle ne peut cependant qu'être limitée tant que les résultats analytiques établis dans un pays ne sont pas admis comme fiables dans les autres pays concernés. Ceci exige que des résultats obtenus en différents lieux puissent être reproduits et comparés, c'est-à-dire obtenus selon des protocoles admis par tous et exprimés dans un langage commun à tous les analystes et utilisateurs. Les organisations nationales et internationales qui s'occupent de métrologie (Tab. I) mènent donc des actions concertées pour tenter de mettre en place une pratique métrologique rigoureuse afin d'assurer la comparabilité des résultats analytiques en chimie. Ces efforts sont cependant encore l'objet de discussions au sein de la communauté des chimistes analystes eux-mêmes, car la mesure en chimie est souvent d'une approche complexe et sectorielle. Elle n'est pas toujours envisageable selon des principes issus de la métrologie en physique et souvent proposés comme modèles. Ceux-ci peuvent apparaître excessivement dogmatiques pour une application en chimie. Il n'en reste pas moins que, fondamentalement, tous les efforts doivent tendre vers la réalisation d'analyses dans le cadre d'un système organisé qui garantisse que les résultats sont exprimés dans un système d'unités reconnu, et fournis aux utilisateurs avec des informations concernant leur signification (intervalle de confiance ou incertitude).

\section{La notion de traçabilité}

La finalité de toute métrologie est la comparabilité des mesures. Elle est donc construite sur la possibilité d'établir une relation de comparaison entre la grandeur à mesurer, le mesurande, et une référence reconnue de même nature; il s'agit de réaliser ce qu'il est convenu d'appeler la traçabilité de résultats de mesurages à des références.

Selon la définition normalisée qui en est donnée dans le Vocabulaire international de métrologie (VIM), « la traçabilité correspond à la possibilité de relier le résultat d'un mesurage (ou de la valeur d'un étalon) à des références déterminées, habituellement des étalons internationaux ou nationaux, par l'intermédiaire d'une chaîne ininterrompue de comparaisons ayant toutes des incertitudes déterminées » [1].

La traçabilité correspond donc à une généralisation de l'étalonnage utilisé par tous dès qu'il faut obtenir un résultat quantifié. Elle apparaît comme une extension indispensable lorsqu'on veut comparer des résultats obtenus avec des étalons d'origines différentes. Une comparabilité effective n'est possible qu'avec des étalons, eux-mêmes étalonnés par 
Tableau I. Liste des organisations concernées par la métrologie en chimie*

$\begin{array}{ll}\text { ISO : } & \text { International Organisation for Standardization. Genève } \\ \text { BIPM : } & \text { Bureau International des Poids et Mesures. Sèvres } \\ \text { CIPM : } & \text { Comité International des Poids et Mesures. Sèvres } \\ \text { CCQM : } & \text { Comité Consultatif pour la Quantité de Matière. Sèvres } \\ \text { CGPM : } & \text { Conférence Générale des Poids et Mesures } \\ \text { CITAC : } & \text { Coopération on International Traceability in Analytical Chemistry } \\ \text { SMT : } & \text { Standard, Measurements and Testing Programme, Commission Européenne. Bruxelles } \\ \text { REMCO : } & \text { ISO Committee for Reference Materials. Genève } \\ \text { NIST : } & \text { National Institute of Standards and Technology. USA } \\ \text { COMAR : } & \text { International Data Bank on Reference Materials, Laboratoire National d'Essais. Paris } \\ \text { CEN : } & \text { Comité Européen de Normalisation. Genève } \\ \text { AFNOR : } & \text { Association Française de Normalisation. Paris } \\ \text { ILAC : } & \text { Internationl Laboratory Accreditation Conference. } \\ \text { EURACHEM : } & \text { European Focus for Analytical Chemistry, Teddington. U.K. } \\ \text { ICH : } & \text { International Conference on Harmonization of Technical Requirements for the Registration of } \\ & \text { Pharmaceuticals for Human Use. Genève } \\ \text { IUPAC : } & \text { International Union of Pure and Applied Chemistry } \\ \text { LNE : } & \text { Laboratoire National d'Essais, Paris } \\ \text { FDA : } & \text { Food and Drug Administration, USA. }\end{array}$

* : d'autres organisations internes à certains pays ne sont pas mentionnées $(A O A C, \ldots)$.

rapport à un étalon d'ordre supérieur, jusqu'à une référence reconnue par tous. Celle-ci, idéalement, sera l'unité de même nature que la grandeur à mesurer dans le Système international d'unités (SI). Le terme traçabilité désigne en premier lieu la propriété du résultat obtenu (ou de la valeur de l'étalon), mais l'usage l'étend aussi au processus permettant d'établir cette propriété. Selon le cas, on dira alors que le résultat du mesurage est traçable à la référence ou on parlera de la traçabilité d'une méthode de mesure si elle permet d'assurer la comparaison entre résultat de mesure et référence. On dira aussi qu'un résultat est traçable à la méthode qui a permis de l'obtenir.

La traçabilité de la mesure et l'évaluation de l'incertitude qui lui est associée sont au centre de toute activité de mesurage dès lors qu'on a besoin d'un résultat analytique indiscutable, utilisable en tout lieu et à tout moment. Ils sont au cœur de tous les efforts pour améliorer la qualité de l'analyse chimique.

\section{Le cas de la chimie : les problèmes rencontrés}

Bien que ce soient des mesures (gravimétriques, volumétriques, etc.) qui ont été à l'origine du développement de la chimie puisqu'elles ont permis de comprendre les interactions entre espèces chimiques et de concevoir les notions de stoechiométrie et de masse moléculaire et atomique, la métrologie, science de la mesure, s'est développée depuis le siècle dernier, surtout dans le domaine des mesures physiques. Ce n'est que récemment, devant l'absence de comparabilité des résultats, divergents quelquefois sur un ou plusieurs ordres de grandeur comme en analyse de traces [2], que les analystes ou les utilisateurs des mesures chimiques ont éprouvé la nécessité de disposer d'un système métrologique rigoureux. Celui-ci devrait idéalement couvrir tous les secteurs socio-économiques où la mesure d'une grandeur chimique est nécessaire, de manière analogue à ce qui existe en physique.

Il y a cependant des raisons qui expliquent le retard pris par les chimistes. En effet, en physique, même s'il existe une grande variété de grandeurs de nature différente, il est toujours possible, et souvent facile, de réaliser une comparaison directe entre la mesure de toutes les grandeurs physiques et un étalon approprié. En chimie, même si des mesurages peuvent porter sur des paramètres physiques (masse, température, intensité de courant...), la grandeur à mesurer est toujours en définitive une quantité de substance, même lorsque le paramètre étudié est de nature physicochimique (vitesse de réaction, constante d'équilibre...). Mais d'une part, il y a un nombre considérable de substances différentes et, d'autre part, une même substance peut exister sous diverses formes. Elle peut être diversement liée à une matrice dans laquelle il est difficile de la distinguer. On conçoit donc les difficultés pour assurer une liaison de traçabilité dans tous les cas entre la substance et un étalon. La comparaison directe au kilogramme ou à la mole de substance demeure exceptionnelle, et la comparaison à des références intermédiaires, correspondant aux échantillons, ne peut être établie que par une méthodologie complexe faisant intervenir une succession d'étapes au cours desquelles il est difficile de maintenir la traçabilité. Ce sont ces difficultés, auxquelles se trouvent confrontés les chimistes analystes, qui ont considérablement freiné le développement d'un 
système de métrologie en chimie et continuent à poser des problèmes. Elles sont augmentées, dans de nombreux pays, par les aspects sectoriels qui régissent souvent les règles de l'analyse chimique. Science servante développée en grande partie pour la caractérisation et l'évaluation de la qualité de matériaux (manufacturés ou naturels), l'analyse en chimie s'est développée, a été réglementée, par secteurs socio-économiques : agro-alimentaire, environnement, médecine, secteurs industriels, justice, armée, etc. Ceci s'est souvent accompagné d'une multiplication des méthodes dites officielles, des étalons de référence, des systèmes de qualité (agréments ministériels, etc.) et des instituts en charge du développement ou de la mise en œuvre des analyses de contrôle.

Même s'il existe un accord sur des principes de métrologie dérivés de la physique, force est de constater qu'ils ne sont pas rigoureusement applicables en chimie. Les efforts et les difficultés pour soumettre les mesures chimiques à ces principes sont à l'origine du retard rencontré dans l'élaboration d'un consensus sur une traçabilité acceptable pour l'ensemble des mesures en chimie. La présentation cidessous s'efforce de résumer les apports des principales contributions et discussions les plus récentes [3-16], issues de groupes de travail créés dans le cadre des organisations internationales s'intéressant à la métrologie en chimie (Tab. I), particulièrement dans le cadre du programme de recherche « Normes, Mesures et Essais » de la Commission Européenne [17]. On examinera jusqu'à quel point il est possible de respecter les principes d'une métrologie rigoureuse. On s'attachera à voir comment on est contraint de développer des procédures fondées sur un consensus plutôt que sur des principes et qui, à défaut de respecter des règles permettant d'obtenir des mesures toujours raccordables aux références SI, cherchent au moins à évaluer les désaccords et les incertitudes.

Les problèmes de représentativité de l'échantillon, qui ne sont pas du strict domaine de la chimie, mais sont aussi de la responsabilité de l'analyste, ne seront pas traités ici bien qu'ils soient souvent à l'origine des plus gros défauts de traçabilité et d'incertitude rencontrés en analyse chimique [18].

\section{Quelle traçabilité en chimie?}

\section{Les références de base, références primaires}

En physique, la traçabilité est réalisée sans ambiguïté par des méthodes simples, comportant un nombre réduit de manipulations. Elles assurent la mesure des grandeurs physiques par la comparaison directe, souvent sans étalonnage, aux unités de base ou dérivées, du Système international. Ces unités constituent les étalons ou références primaires, bases de la traçabilité [1].

En chimie, il s'agit de mesurer dans un échantillon une quantité de composés, atomes, ions, molécules ou édifices moléculaires. Deux unités SI principalement sont dispo- nibles, le kilogramme, unité de masse et la mole, unité de quantité de substance [1]. L'objet de l'analyse en chimie est de déterminer des associations d'espèces élémentaires, des compositions de mélanges de substances définies ou des produits de réactions mettant en jeu des nombres de moles. La mole, représentative de ces entités chimiques, est de ce fait considérée depuis la $14{ }^{\text {ème }}$ Conférence Générale des Poids et Mesures en 1971 [19] comme l'unité SI de quantité de substance. La mole est la quantité de substance contenant autant d'entités élémentaires de la substance qu'il y a d'atomes dans 0,012 $\mathrm{kg}$ de l'isotope 12 du carbone. Elle est réellement l'unité universelle, référence de base pour tous les composés chimiques. Elle est cependant particulière en ce que la définition d'une mole de substance spécifie la nature de l'entité chimique à laquelle elle correspond. Elle n'est pas matérialisée physiquement par un étalon contrairement au kilogramme.

La traçabilité d'une quantité de substance pure au kilogramme est réalisable directement, par pesée par exemple. La traçabilité à la mole n'est pas toujours réalisable directement. Cependant mole de substance et kilogramme sont reliés par un facteur de proportionnalité caractéristique de chaque composé, sa masse atomique ou moléculaire qui est l'intermédiaire permettant une traçabilité secondaire à la mole. Pour certaines substances qui possèdent des propriétés physico-chimiques moléculaires particulières, il existe des méthodes qui permettent d'obtenir une traçabilité directe à la mole (voir ci-dessous). Ce peut-être par exemple le cas de composés électroactifs traçables à la mole par coulométrie. L'utilisation de l'une ou l'autre de ces deux unités fondamentales est souvent une question de circonstance, de commodité ou d'habitude.

Les unités SI sont donc, dans un système métrologique idéal tel qu'en physique, les références ou étalons primaires auxquels toute mesure doit pouvoir être traçable. La mise en œuvre se fait au moyen d'étalons intermédiaires, nationaux, locaux, de même nature, qui leur sont directement comparables. Ces étalons sont habituellement conservés dans chaque pays par les organismes nationaux de métrologie. Ils se situent au niveau du premier maillon de la chaîne de traçabilité. En chimie, ce sont les unités SI, mole et kilogramme qui sont les références primaires. Les références pratiques matérialisées ne peuvent être que des kilogrammes étalons locaux (Fig. 1).

\section{Les méthodes primaires: quelle utilisation en chimie?}

La traçabilité au kilogramme ou à la mole telle qu'elle est définie ci-dessus est réalisable pour les substances pures par gravimétrie par exemple. Lorsque le composé d'intérêt ne peut être complètement séparé ou n'est pas accessible totalement de manière spécifique dans son milieu, la gravimétrie est inutilisable. Dans ce cas, il existe un certain nombre de méthodes qui peuvent être utilisées en chimie pour évaluer directement la quantité de substance et possèdent les caractéristiques d'une méthode de mesure primaire. Selon la définition adoptée en 1985 par le BIPM, à la suite de la 


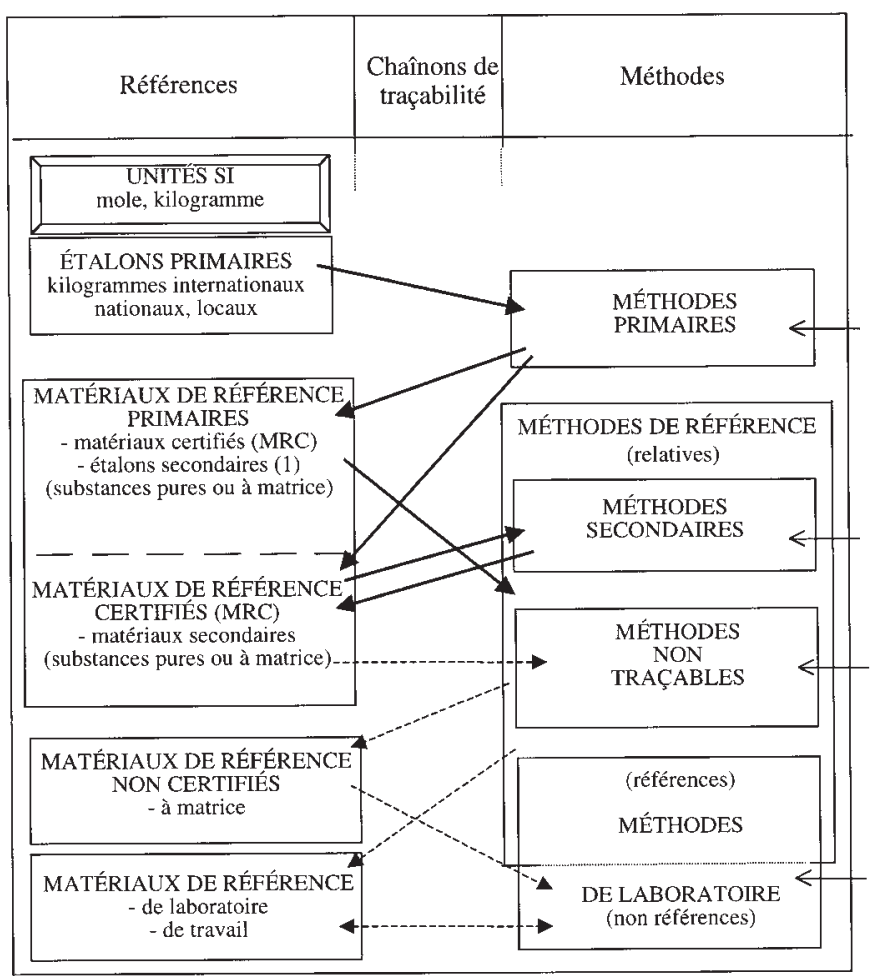

Figure 1. Hiérarchie des outils nécessaires à la traçabilité en chimie. $\longrightarrow$ : chaîne de raccordement à traçabilité assurée. $---->$ : chaînon à traçabilité incertaine. $\longrightarrow$ : entrée des échantillons.

première réunion du Comité Consultatif pour la Quantité de Matière (CCQM) (Tab. I) [20],

- une méthode primaire est une méthode qui possède les plus hautes qualités métrologiques;

- dont la mise en ceuvre peut être entièrement décrite par une équation reliant la mesure à la quantité de substance ;

- pour laquelle une incertitude exprimée en unité SI peut être entièrement évaluée, en particulier en ce qui concerne les contributions éventuelles dépendant d'autres substances ou de la matrice contenant la substance;

- dont les résultats sont donc obtenus sans référence à un étalon.

Les méthodes répondant à cette définition, proposées par le CCQM (Tab. I), sont cependant en nombre très limité. Ce sont essentiellement : la coulométrie, la gravimétrie, la titrimétrie, la dilution isotopique avec spectrométrie de masse (IDMS), la mesure de l'abaissement du point de congélation.
Si l'on prend comme exemple la coulométrie, on a bien en effet l'équation décrivant la mesure $\frac{I \cdot t}{z \cdot F}=n$ qui relie directement la mesure de l'intensité $I$ et du temps $t$ au nombre de mole $n$ d'une substance électrolysable de nombre de charge $z$ et, si l'on exprime les grandeurs mesurée $I$ et $t$ et la constante $F$ en unités SI, respectivement en ampère, seconde et coulomb/mole, $n$ est obtenu en mole, de même que l'incertitude sur la mesure.

Une méthode primaire est donc spécifique pour une espèce chimique et la relation entre la mesure et la quantité de substance doit être indépendante ou corrigible de toute interférence. Les exemples d'application de ces méthodes à des problèmes analytiques courants sont cependant extrêmement rares : la mesure d'une masse moléculaire par abaissement du point de congélation est certainement devenue une curiosité de laboratoire. Même l'utilisation de la gravimétrie, pourtant à l'origine de la mesure en analyse chimique, n'est plus pratiquée que de manière très spécifique dans les laboratoires d'analyse.

L'IDMS est souvent présentée comme une méthode qui serait plus largement utilisable. Elle est basée sur l'addition à l'échantillon d'une quantité connue du composé d'intérêt, sous une forme ayant subi un enrichissement isotopique, utilisé comme étalon interne. La teneur en substance analysée est déduite de la mesure des rapports isotopiques pour l'échantillon, l'étalon et leur mélange. La méthode, ne faisant intervenir que des mesures de masse et de rapports isotopiques reliés à la mole et déterminés avec une faible incertitude est quelquefois considérée comme ayant le potentiel d'une méthode primaire $[9,21,22]$. La mesure est cependant réalisée par l'intermédiaire d'un étalon interne et la méthode ne peut être considérée comme définitive que si la mesure des rapports isotopiques est pratiquement indépendante de la référence. Le protocole opératoire est alors très rigoureux et complexe et exige que l'on puisse disposer de références, enrichies et non enrichies, parfaitement caractérisées. Il faut s'assurer que les incertitudes apportées par le processus d'étalonnage et la technique d'ionisation son négligeables $[22,23]$. Dans ces conditions, et pour un certain nombre d'analyses de substances simples (analyse d'éléments inorganiques pluriisotopiques), des résultats ont effectivement été obtenus avec une faible incertitude et la méthode a été proposée comme référence [23]. Mais dans le cas général d'échantillons à matrice, contenant des composés interférents mal connus ou présents en quantités majeures (analyse de traces dans les métaux), des prétraitements modifiant ou détruisant la substance à doser sont nécessaires. Les causes d'incertitude et les défauts de traçabilité sont alors les mêmes que pour les méthodes physicochimiques usuelles. Les difficultés de mise en œuvre et le coût d'utilisation de l'IDMS lui font préférer d'autres méthodes réellement non primaires mais plus économiques.

D'autres méthodes peuvent aussi être proposées comme méthodes primaires potentielles. C'est le cas de l'analyse par activation neutronique. Elle est cependant le plus souvent 
utilisée comme méthode relative, et considérée alors comme méthode de référence pour l'analyse de traces de certains éléments. Néanmoins, il existe peu de domaines ou d'échantillons en chimie où les méthodes primaires peuvent être utilisées avec un avantage assuré par rapport aux méthodes relatives usuelles. Il n'est pas évident qu'elles fournissent nécessairement les résultats les plus justes et les moins incertains.

\section{Les méthodes d'analyse au laboratoire}

\section{Les méthodes traçables secondaires}

Dans la réalité quotidienne d'un laboratoire d'analyse en chimie, des difficultés rédhibitoires empêchent souvent que l'on puisse établir une traçabilité absolue. Compte tenu de la complexité des échantillons, la substance à analyser est difficilement isolable ou accessible dans sa matrice. Sa mesure est exceptionnellement traçable par une méthode primaire. L'analyse chimique est généralement réalisée par tout ou partie d'une succession d'étapes au cours desquelles l'échantillon initial est physiquement détruit (par fusion, digestion, calcination), mis en solution, puis le composé d'intérêt, ou son transformé, est soumis à extraction, séparation, prétraitement. Finalement, il est détecté et quantifié par des méthodes spectroscopiques ou électrochimiques dont le signal est transmis à un calculateur qui le traite et fournit le résultat. La traçabilité ne peut être réalisée que si, au cours de la succession des différentes étapes, une comparaison entre l'espèce analysée et une référence de même nature peut être maintenue (Fig. 2). Il faut aussi qu'une évaluation de l'incertitude soit obtenue simultanément.

Les méthodes physico-chimiques d'analyse sont basées sur des lois physiques décrivant les interactions d'un rayonnement, d'un potentiel électrique, de la température avec la matière et mesurent la variation résultante d'une grandeur physique (rayonnement secondaire, émission de particules, courant électrique...). Lorsqu'une telle grandeur est une propriété caractéristique d'une substance, sa mesure permet d'établir une relation directe avec la quantité de substance, généralement exprimée en nombre de moles ou en concentration (mole par masse ou par mole d'échantillon). Ces lois établies à partir de systèmes simples ne sont cependant applicables que de manière approximative à la plupart des systèmes complexes que sont les échantillons de la chimie. Les effets de matrice d'autres facteurs que les caractéristiques physiques ou chimiques de la substance analysée influent sur le signal. Les méthodes physico-chimiques ne peuvent donc que rarement être des méthodes primaires malgré les efforts réalisés pour évaluer des réponses spécifiques pour des espèces chimiques particulières [24].

Pour assurer la traçabilité, un étalonnage est réalisé en comparant le signal mesuré obtenu avec la substance étudiée, au signal obtenu, dans des conditions rigoureusement identiques, avec une quantité déterminée de substance de référence (Fig. 2). Cela suppose aussi que le composé analysé puisse être intégralement isolé de sa matrice ou que le signal obtenu soit insensible à la matrice. Les méthodes de

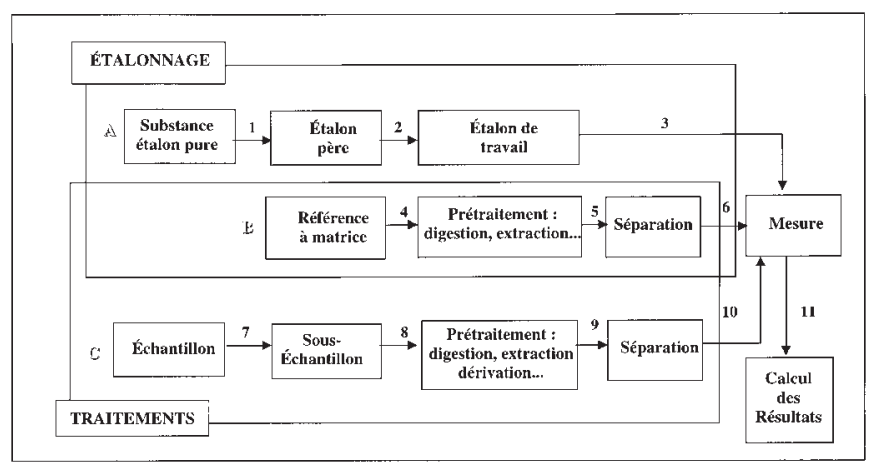

Figure 2. Schéma des étapes d'une procédure analytique en chimie : A : Chaîne d'étalonnage avec MR substance pure, B : Chaîne d'étalonnage et traitements possibles dans le cas de MR à matrice (analyse élémentaire...). C : Chaîne des traitements possibles pour l'échantillon. Les numéros correspondent aux maillons de traçabilité entre les étapes. (voir aussi Tab. II).

l'analyse chimique sont donc souvent constituées de plusieurs techniques couplées, mais elles sont traçables si l'on prend toutes les précautions pour assurer la rigueur de l'étalonnage, si aucune erreur systématique n'affecte les différentes étapes, et si l'incertitude reste suffisamment faible tout au long du processus analytique (voir ci-dessous). Ces méthodes sont qualifiées de relatives pour les distinguer des méthodes primaires considérées comme définitives ou absolues (Fig. 1).

Cette situation idéale est celle que l'on rencontre par exemple en analyse élémentaire inorganique avec une méthode telle que l'ionisation par plasma (ICP) couplée à la spectrométrie d'émission atomique ou à la spectrométrie de masse. Après la mise en solution complète de l'échantillon, la traçabilité de la mesure de la quantité d'un élément doit être assurée par un étalonnage au moyen d'une solution de référence certifiée (voir ci-dessous). En s'assurant qu'aucune interférence ne fausse la mesure, l'analyse est alors réalisée par une méthode non primaire, mais traçable à une référence indiscutable.

\section{Quelles méthodes en l'absence de traçabilité}

Dans beaucoup de cas, compte tenu des nombreuses étapes de l'analyse, au cours desquelles l'état physico-chimique de l'échantillon ou du matériau de référence sont modifiés, il n'est pas possible d'assurer des liaisons de traçabilité indiscutables. Sur la figure 2 sont schématisées ces étapes pour l'étalonnage et les traitements de l'échantillon avant la mesure. On doit distinguer l'étalonnage simple pour l'analyse d'une substance pure, et l'étalonnage pour une substance à matrice où le matériau de référence subit les mêmes traitements, de façon à neutraliser les interférences éventuelles provenant de la matrice. Dans le tableau II sont proposés les points d'interruption possibles de la traçabilité pour différents types d'échantillons. L'exemple le plus 
Tableau II. Niveaux d'interruption de la traçabilité et outils permettant d'assurer la comparabilité, selon le type d'échantillon, en référence à la figure 2. Les nombres correspondent aux chaînons de traçabilité de la figure 1. Ils sont entre parenthèses lorsque la traçabilité peut être assurée, sans parenthèse lorsque la traçabilité est interrompue. MP : Méthode primaire, MST : Méthode secondaire traçable, MNTV : méthode non traçable validée, ML : méthode de laboratoire (voir Fig. 1).

\begin{tabular}{|c|c|c|c|c|c|}
\hline \multirow{2}{*}{$\begin{array}{l}N^{\circ} \text { de Chaîne } \\
\text { (Fig. 1) }\end{array}$} & \multicolumn{5}{|c|}{ Nature des échantillons } \\
\hline & Inorganique & Gazeux & Organique & Organo-métalliques & Biochimique \\
\hline A & (3) & (3) & (1) (3) & $1(3)$ & \\
\hline B & (4) (5) (6) & (7) (10) & $4,5(6)$ & $4,5(6)$ & $4,5(6)$ \\
\hline \multirow[t]{3}{*}{ C } & (7) (8) (9) (10) & (7) $8,9(10)$ & (7) $8,9(10)$ & (7) $8,9(10)$ & \\
\hline & $(11)$ & (11) & (11) & (11) & (11) \\
\hline & \multicolumn{5}{|c|}{ Outils nécessaires pour réaliser la comparabilité } \\
\hline Matériau* & MRP, MRC & & MRP, MRC & MRC, MR & MRC, MR \\
\hline Méthode & MP, MST & MP & MST, MNTV & MST, MNTV, ML & MST, MNTV, ML \\
\hline
\end{tabular}

* : sigles en référence au texte.

représentatif de telles ruptures est sans doute celui où une extraction est nécessaire : pour obtenir un résultat traçable, il faudrait pouvoir assurer que l'extraction est totale ou au moins répétable et déterminer les pertes. Tous les chimistes analystes savent que ce n'est pratiquement jamais possible. On a rarement une connaissance sûre de la valeur du rendement de l'extraction ; en effet il n'existe que très rarement des matériaux de référence, à matrice identique à celle de l'échantillon, permettant d'évaluer la qualité de l'extraction. Par ailleurs, on n'a jamais l'assurance que dans le matériau de référence à matrice dont on dispose, naturel ou dopé, la substance soit intégralement sous une forme chimique comparable à celle du composé à analyser dans l'échantillon réel, à supposer qu'il soit sous une seule forme. Les méthodes d'extraction sont toujours le maillon faible de toute traçabilité en analyse chimique, du moins si l'on ne tient pas compte de l'échantillonnage.

Devant ces difficultés illustrant l'impossibilité de bâtir en chimie un système métrologique simple et rigoureux, un consensus s'est établi pour élaborer des solutions pragmatiques en utilisant des méthodes « métrologiquement acceptables » qui permettent, en l'absence d'une traçabilité absolue, d'assurer au moins une comparabilité des résultats. Il s'agira toujours de méthodes :

- utilisées dans un but analytique bien précis ;

- bien définies et décrites en détail ;

- appliquées à la mesure d'un seul ou d'une famille de composés d'intérêt ;

- mises en œuvre selon des critères de validation rigoureux.

Le défaut de traçabilité peut interdire une évaluation de l'incertitude par rapport à une valeur « absolue ». Ces méthodes ne peuvent être que des méthodes secondaires (Fig. 1). Elles peuvent cependant être proposées comme méthodes de références, normalisées ou non, pour des analyses spécifiques bien définies, si elles peuvent être validées dans les conditions présentées ci-dessous.

\section{Validation d'une méthode d'analyse}

Le but de toute méthode d'analyse est d'apporter des éléments de réponse (les résultats analytiques) à un problème analytique particulier. La validation est l'opération par laquelle on s'assure que ces résultats répondent au problème de manière satisfaisante pour l'utilisateur. Elle s'efforce de détecter et contrôler les sources d'erreurs possibles liées à la méthode; une définition générale en est proposée par la FDA (Tab. I) : "valider une méthode consiste à démontrer, avec un degré de confiance élevé et sous une forme documentée, que la méthode permet d'obtenir un résultat analytique qui atteint les spécifications définies à l'avance ».

C'est sans doute actuellement dans le domaine de l'analyse de radioactivité et de l'analyse pharmaceutique que les recommandations les plus élaborées concernant la validation des procédures analytiques ont été publiées [25,26]. La validation est un processus en plusieurs étapes [25,27]. Au niveau du laboratoire, une fois établi le choix de la méthode avec les différentes opérations analytiques successives nécessaires, elle consiste en une évaluation interne qui comprend une suite d'études expérimentales et statistiques permettant de réaliser :

- la détermination des caractéristiques de la méthode : sélectivité, domaine de linéarité, sensibilité, limites de détection et de quantification, robustesse ; 
- l'étalonnage pour lequel il faut d'ailleurs distinguer « l'étalonnage de l'appareillage » qui correspond au test du paramètre physique mesuré par l'appareil et «l'étalonnage de la méthode » qui permet d'établir la relation signal-quantité de substance; ce dernier doit être réalisé avec des étalons et des matériaux de références adaptés, tenant compte de la matrice autant que possible ;

- la recherche des interférences provenant des impuretés de l'échantillon ou des matériaux de référence, et de la méthode d'analyse elle-même ;

- l'évaluation des incertitudes globales et composantes, en particulier concernant des rendements d'extraction s'il y a lieu (voir ci-dessous).

La validation se poursuit par une évaluation des résultats obtenus par la méthode pour l'analyse d'un matériau de référence certifié, de manière interne au laboratoire. L'idéal est cependant de tester la méthode au cours d'analyses interlaboratoires, qui permettent de vérifier si l'incertitude obtenue correspond au domaine de variabilité généralement accepté. L'étalonnage est un des éléments clés en ce qu'il établit, pratiquement, la traçabilité de la méthode. L'utilisation d'une substance de référence pure de l'entité analysée n'est pas suffisante : l'étude des interférences doit être faite en utilisant des blancs, des échantillons avec ajouts et des matériaux à matrice. Les étalonnages réalisés au niveau des différentes étapes de l'analyse assurent la traçabilité du maillon correspondant, mais c'est l'étalonnage global de la méthode dans son ensemble qui doit finalement être réalisé.

Dans le cas où l'on dispose d'un matériau de référence, éventuellement à matrice, on réalise un étalonnage externe. Si l'on ne dispose pas de matériau de référence, on réalise un étalonnage interne par addition d'un standard interne, ou par la méthode des ajouts dosés lorsqu'on cherche à corriger des interférences dues à la matrice [27, 28].

Une méthode ne peut être validée que pour une analyse avec des spécifications bien précisées, indiquant par exemple le type de matrice, les gammes de concentration en substance analysée, les niveaux d'incertitude acceptés et éventuellement les conditions d'emploi, si elles risquent d'être modifiées en cours d'essais. La validation d'une méthode, et donc l'usage de la méthode comme référence, ne sont jamais établis de façon définitive ; il faut renouveler les contrôles de façon suivie pour s'assurer qu'il n'y a pas de déviation au cours du temps.

\section{Les matériaux de référence}

\section{Définition}

En parallèle avec les méthodes de références, les matériaux de référence constituent le deuxième support de la traçabilité en chimie. Ils sont utilisés comme étalons, outils de transfert de la traçabilité et pour assurer le contrôle des performances des méthodes d'analyse. Ils sont définis par des normes internationales [1] et les informations concernant leur élaboration et leur certification sont présentées dans des guides publiés par l'ISO [29] et le BCR [30] et détaillées dans des publications récentes [31,33].
Un matériau de référence (MR) est un matériau ou une substance dont une ou plusieurs valeurs de ses propriétés sont suffisamment bien établies pour permettre de l'utiliser pour l'étalonnage d'un appareil, l'évaluation d'une méthode de mesurage ou l'attribution de valeurs à des matériaux.

Un matériau de référence est certifié (MRC) si une ou plusieurs valeurs de ses propriétés sont certifiées par une procédure qui établit son raccordement à une réalisation exacte de l'unité dans laquelle les valeurs des propriétés sont exprimées et pour laquelle chaque valeur certifiée est accompagnée d'une incertitude à un niveau de confiance indiqué. Il est accompagné d'un certificat.

En chimie, les propriétés des matériaux de référence sont la composition ou la teneur en une ou plusieurs substances ou entités chimiques, exprimées en nombres de moles et/ou unités de masse. La différence entre MR et MRC réside essentiellement dans le niveau de fiabilité qu'on reconnaît aux valeurs de ces teneurs. Pour les MRC on s'efforce de réaliser la traçabilité de la teneur certifiée à la mole ou au kilogramme. Ceci n'est pas exigé pour les MR pour lesquels on peut accepter aussi une incertitude plus grande et moins bien définie. Mais dans les deux cas un très haut niveau d'homogénéité et de stabilité à long terme est exigé pour limiter les incertitudes liées à des variations de ces deux critères.

\section{Les matériaux de référence primaires}

La caractéristique des méthodes primaires est qu'elles permettent d'établir le maillon initial d'une chaîne de traçabilité, rarement suffisant pour réaliser une analyse chimique. Elles permettent en particulier, à partir des étalons primaires, d'obtenir les étalons secondaires [1] qui, en chimie, constituent des matériaux de référence qu'il est convenu d'appeler les matériaux de référence primaires (MRP) selon la définition adoptée par le CIPM (Tab. I) en 1995 :

«Un matériau de référence primaire est un matériau possédant les plus hautes qualités métrologiques, dont la valeur est déterminée par une méthode primaire de mesure. »

Il s'en suit donc qu'un MRP est mesuré par une méthode qui lui est spécifique et qui doit aussi permettre de connaître et évaluer les corrections éventuelles qui dépendent d'autres espèces ou de la matrice, avec une incertitude appropriée. Un MRP possède donc par nature la qualité de matériau de référence certifié. Il ne doit pas être confondu avec les références primaires définies précédemment et identifiables aux unités SI (Fig. 1).

Le nombre des MRP disponibles et adaptés à la multiplicité et à la complexité des analyses est cependant tout à fait insuffisant. On est donc conduit à élaborer d'autres matériaux de référence, pour s'efforcer de répondre à l'ensemble des besoins en analyse chimique.

\section{Élaboration des matériaux de référence}

Les MR peuvent être des matériaux synthétiques ou des échantillons naturels. Leur élaboration doit respecter des 
exigences très rigoureuses qui sont souvent difficiles à atteindre [32]. En particulier lorsqu'il s'agit de matériaux à matrice, la référence doit être comparable à l'échantillon autant que possible. On doit s'efforcer de connaître l'état physique du matériau, son homogénéité, la composition de la matrice, la concentration des substances d'intérêt, leurs formes chimiques, la nature de leurs liaisons physico-chimiques avec la matrice et les interférences possibles.

Les principes généraux à respecter pour la certification des MR et les méthodologies à utiliser suivant le paramètre à certifier sont présentés dans le guide ISO 35 [29]. Dans certains cas une seule méthode primaire utilisée par un seul laboratoire, telle que l'IDMS, considérée comme « définitive » peut être suffisante pour assurer une certification [23]. Cependant, indépendamment du fait que les méthodes absolues sont peu nombreuses et d'application très restreinte, le manque de contrôle des biais éventuels fait préférer une approche basée sur l'utilisation de plusieurs méthodes indépendantes, dans des laboratoires différents [31]. Dans ce cas, lorsque des résultats comparables sont obtenus par plusieurs laboratoires, les conditions sont réunies pour éviter les erreurs systématiques et permettre la certification du matériau. En particulier, les rendements des extractions et des pré-traitements doivent être évalués. Nous avons vu que si l'on est confronté à des échantillons complexes, il n'est pas toujours possible d'assurer la traçabilité tout au long du processus analytique. C'est par exemple le cas pour l'élaboration de MR à partir de matériaux d'origine naturelle comme des tissus d'animaux contenant des substances toxiques. Si l'incertitude associée à l'ensemble des mesures est jugée acceptable, le matériau peut être certifié ; la valeur moyenne des résultats et l'incertitude associée constituent la valeur certifiée. C'est la démarche adoptée par le Programme SMT pour l'élaboration des MRC et MR du BCR.

Les matériaux non certifiés sont obtenus avec des exigences moindres concernant les valeurs déclarées. Ils peuvent être élaborés par un seul laboratoire utilisant une méthode validée et bien contrôlée, ou mieux, par plusieurs méthodes différentes de façon à évaluer l'incertitude de manière plus complète. Ils peuvent aussi être le résultat de campagnes interlaboratoires de certification n'ayant pas permis d'atteindre les critères d'incertitude exigés pour la certification.

\section{Utilisation des matériaux de référence [31-33]}

Les MRC sont une des voies de l'analyse vers l'exactitude. Ce sont soit des substances pures ou des solutions de composés bien définis, soit des matériaux à matrices, utilisables en principe seulement pour des analyses bien spécifiques. Ils assurent la traçabilité des processus de mesure aux références internationales, nationales, locales. Ils servent à fixer la valeur d'étalons de travail de même nature utilisés comme référence à usage interne pour un ou plusieurs laboratoires. Ils permettent la validation des méthodes plus rapidement que par la comparaison avec les résultats obtenus par une méthode de référence. Ils sont essentiels pour vérifier les performances d'une méthode nouvellement installée.
Compte tenu de leur coût, ils sont généralement réservés pour les tests ultimes de qualification des procédures d'analyse.

Pour les MR il y a une exigence d'exactitude moindre que pour les MRC. Ils peuvent être des produits purs ou des solutions de composition connue utilisés pour tester des méthodes en analyse inorganique ou pour évaluer les différentes procédures techniques mises en œuvre au cours d'analyses en plusieurs étapes (extraction, prétraitement, séparation...), fréquentes en analyse organique ou de spéciation. Ils peuvent être des matériaux de référence internes à un laboratoire, obtenus par comparaison avec un MRC, spécifiques pour une analyse particulière, ayant une teneur connue en composé d'intérêt, avec une matrice aussi proche que possible de celle de l'échantillon à analyser. Ils peuvent aussi être utilisés pour comparer les performances de techniques analytiques et ils sont indispensables pour tester la reproductibilité des mesures et la dérive en analyse de routine, en particulier pour les diagrammes de contrôle. Ils peuvent servir à évaluer les performances de laboratoires au cours d'intercomparaisons.

À partir de l'usage des différents types de références, on peut envisager, en rejoignant les propositions de quelques auteurs [10,14,34] et dans le cadre d'un schéma de la traçabilité en chimie, une hiérarchie de l'ensemble de ces références présentée sur la figure 1. Le sommet est représenté par les références primaires que sont les unités SI, mole et kilogramme étalon. En chimie les seuls étalons nationaux et locaux qui peuvent ensuite leur être directement reliés sont des kilogrammes. Ce sont là des références ou étalons primaires qui constituent pour les laboratoires le point d'ancrage de l'étalonnage des balances et assurent la traçabilité des mesures de masse (et de nombre de moles). Viennent ensuite les matériaux de référence pour l'étalonnage des méthodes, parmi lesquels on peut distinguer :

- les matériaux de référence primaire (MRP), étalons secondaires de la quantité de substance [1], constitués de substances pures et de quelques rares matériaux à matrices, traçables aux références supérieures par des méthodes primaires, et dont la valeur de référence est certifiée :

- les matériaux de référence certifiés (MRC), matériaux de référence obtenus par une méthode traçable ; ce sont des substances pures, des mélanges ou solutions ou des matériaux à matrice ;

- les matériaux de référence à matrice, non certifiés (MR) dont la composition de la matrice est incomplètement connue ou dont la teneur ne peut pas être tracée rigoureusement à une référence certifiée ; ils peuvent cependant être reconnus comme références, faute de mieux, si la valeur qui leur est attribuée est obtenue à la suite de tests d'intercomparaison et correspond à la moyenne statistique des résultats, associée à une incertitude définie ; cette valeur d'usage peut être acceptée de manière consensuelle comme valeur certifiée ; 
- les matériaux de références de laboratoire, à traçabilité non établie et à usage interne et limité.

L'incertitude des valeurs attribuées aux différents niveaux de référence ne devrait pas influer de manière significative sur l'incertitude du niveau suivant. Elle augmente évidemment avec l'éloignement des références primaires. Elle doit cependant au terme de l'analyse, être suffisamment inférieure à l'incertitude demandée pour la mesure à réaliser afin d'assurer que le résultat est pleinement exploitable par l'utilisateur.

Parallèlement aux différents niveaux de matériaux de références on peut envisager aussi, comme cela est représenté sur la figure 1, un positionnement des différents niveaux de méthodes de mesure. Depuis les unités SI via les étalons primaires et les MR, jusqu'aux substances à analyser, sont figurées les liaisons possibles entre références et méthodes. La qualité des maillons de la chaîne de traçabilité évolue suivant la hiérarchie des références et des méthodes. La longueur de la chaîne de traçabilité augmente généralement avec la complexité de l'échantillon. Suivant leur nature, leur état physique, leur complexité, l'entité chimique à déterminer, l'usage que l'on peut faire du résultat de la mesure, les échantillons seront analysés au niveau de la méthode la mieux adaptée.

\section{Disponibilité des matériaux de référence}

Pour répondre à une demande croissante en analyses fiables, il est nécessaire de disposer d'un nombre croissant de MR et en particulier de MRC dans les différents domaines de l'analyse chimique. L'élaboration de ces matériaux est longue et coûteuse et leur disponibilité en quantité suffisante ainsi que leur conservation sur le long terme doivent être assurées. Ces exigences ne peuvent être réalisées que par des organismes nationaux ou internationaux qui s'efforcent de couvrir divers domaines d'application de l'analyse. Ce sont souvent ces mêmes organismes qui sont à l'origine de l'élaboration des MRC et qui organisent les campagnes interlaboratoires nécessaires pour aboutir à leur certification.

Il existe un grand nombre de fournisseurs de MR et MRC selon les différents domaines d'analyses. Deux organismes importants, le National Institute of Science and Technology (NIST) aux États-Unis et le BCR de la Commission Européenne couvrent divers domaines et assurent la disponibilité des matériaux sur le long terme. L'IAEA, International Atomic Energy Agency à Vienne (Autriche) fournit surtout des matériaux certifiés pour les mesures nucléaires ou isotopiques et le National Institute for Environmental Studies (NIES) au Japon des matériaux pour l'analyse environnementale. Le Council on Reference Materials de l'ISO (REMCO) (Tab. I) publie un annuaire des matériaux de référence [35]. L'IUPAC a été le premier organisme à publier un catalogue des MRC disponibles [36] et il existe des compilations dans des domaines spécialisés [4,37]. Une source d'informations très importante est la banque de références du COMAR consultable au Laboratoire National d'Essais (Tab. I).

\section{L'incertitude de la mesure. Comment l'évaluer?}

La valeur vraie d'un mesurande ne peut jamais être connue, elle peut seulement être approchée. Pour toutes les raisons rappelées ci-dessus, c'est particulièrement vrai pour la mesure d'une quantité de substance. Les études de Horwitz sur la variabilité des mesures en fonction de la concentration $[2,38]$ confirment cet état de fait. La confiance que l'on peut accorder à un résultat analytique ne peut être évaluée que si on lui associe une estimation de l'étendue des valeurs dans laquelle se situe la vraie valeur de la grandeur mesurée. C'est l'incertitude sur la mesure. La définition en est donnée dans le VIM [1] : «paramètre associé au résultat d'un mesurage, qui caractérise la dispersion des valeurs qui pourraient raisonnablement être attribuées au mesurande ».

Cependant, pour pouvoir comparer les incertitudes annoncées, il est impératif que les méthodes d'évaluation et les modes d'expression des incertitudes soient uniformes. Le paramètre retenu pour représenter l'incertitude est généralement l'écart-type, obtenu à partir de la distribution statistique de résultats correspondant à des séries de mesurages. Des recommandations générales, quel que soit le domaine de mesure, ont été élaborées par un groupe de travail du BIPM et sont présentées dans un guide ISO [39], et reprises par un guide EURACHEM [40] pour ce qui concerne l'analyse chimique.

Dans le cas d'une analyse chimique, des incertitudes sont apportées à chaque étape d'un mesurage généralement complexe et ont des causes très diverses : mauvaise représentativité ou dégradation de l'échantillon, perte au cours des extractions ou préconcentrations, effets de matrice et interférences méconnues au cours des diverses opérations analytiques, biais introduits par la technique d'analyse ou l'opérateur, incertitudes sur la valeur des étalons ou des matériaux de référence et sur les constantes et autres paramètres utilisés dans l'algorithme du calcul. On sait par exemple que l'incertitude relative sur la masse moléculaire des éléments est presque toujours très inférieure à $10^{-4}[41,42]$ et qu'elle est donc négligeable dans la plupart des analyses courantes par comparaison aux composantes expérimentales. Ces incertitudes sont une indication de la qualité de la relation de traçabilité du chaînon de mesurage que constitue chaque étape.

L'approche recommandée pour évaluer l'incertitude consiste à évaluer ou estimer chacune de ces incertitudes composantes et à les combiner pour obtenir l'incertitude globale [39, 40]. L'intérêt d'une telle décomposition est sans doute d'aider à déterminer au mieux les différentes sources d'erreurs qui peuvent apparaître au cours du processus analytique et d'en permettre le contrôle. Mais cette approche, envisagée pour une méthodologie idéale, n'est possible en chimie que pour des analyses d'échantillons simples par des méthodes primaires par exemple, où dans le cas de méthodes ne comprenant que peu d'étapes bien contrôlables. Elle n'est pas intégralement applicable dans beaucoup d'analyses où 
l'incertitude associée à certaines étapes n'est pas accessible. $\mathrm{Ce}$ cas se rencontre lorsque les matrices de l'échantillon sont complexes, et d'une manière générale, lorsqu'une des étapes du processus analytique ne permet pas d'assurer la traçabilité. Dans ce cas, l'estimation des erreurs « d'après l'expérience acquise ou d'après d'autres informations », selon les indications du guide ISO, semble une recommandation bien peu rigoureuse. Ce type d'approche est donc souvent complexe à mettre en œuvre et peut exiger un travail de longue durée, souvent incompatible avec les délais exigés dans des laboratoires d'essais et le budget des demandeurs.

Une procédure alternative consiste à évaluer l'incertitude sur le résultat analytique à partir de mesures répétées obtenues par la mise en œuvre de l'ensemble du processus analytique, par plusieurs laboratoires, puis par l'exploitation statistique de l'ensemble des résultats obtenus $[43,44]$. Une telle détermination globale est d'un abord plus simple que l'approche précédente, avec laquelle elle est néanmoins compatible puisqu'elle inclut mais sans les distinguer, les incertitudes correspondant à toutes les erreurs systématiques ou variations aléatoires provenant des méthodes ou des analystes. Elle inclut en particulier, sans qu'il soit nécessaire de les « estimer », les incertitudes non évaluables. Elle exige l'organisation d'essais interlaboratoires et ne permet pas de contrôler les étapes ni les acteurs de l'analyse, mais elle est adaptée aux analyses complexes tant que les incertitudes évaluées sont acceptables, c'est-à-dire compatibles avec les demandes des utilisateurs.

Si l'incertitude globale est jugée trop élevée, il est alors nécessaire d'en rechercher la raison par un réexamen des étapes individuelles les plus influentes, même réalisé de façon partielle. Un protocole opératoire analogue à celui utilisé au cours de la validation est alors applicable. Par exemple, des solutions simples seront utilisées pour tester la détection des extraits bruts pour la purification, des extraits purifiés pour les dérivations et les séparations, des échantillons enrichis (faute de mieux) pour l'extraction, et des échantillons réels certifiés pour évaluer la procédure dans son ensemble. Si possible, ceci devrait se faire en comparaison avec d'autres laboratoires. C'est le type de procédure utilisé au BCR pour l'évaluation des méthodes au cours des études interlaboratoires [33, 45]. L'intercomparaison consiste en une revalidation interlaboratoire des méthodes. Les tests de répétabilité et de reproductibilité [1] et une exploitation statistique des résultats peuvent permettre de distinguer les composantes associées à la méthode, au laboratoire, à l'essai lui-même, ou celles qui sont aléatoires [17, 43]

L'incertitude est une propriété liée à un traitement statistique des résultats analytiques, et c'est une information essentielle pour évaluer la qualité d'une mesure. Elle est en effet le moyen de contrôle du processus analytique dont elle permet de déceler les points faibles. Son évaluation impose à l'analyste une critique permanente de son travail et des procédures qu'il utilise. Elle le pousse à mettre en pratique les moyens qui garantissent la fiabilité de ses résultats et au besoin conduisent à leur amélioration, c'est-à-dire contrôle qualité et assurance qualité. Elle lui permet de fournir à l'uti- lisateur des résultats qui constituent une base de décision objective. Toutefois, il faut rester conscient que la première qualité d'un résultat analytique est son adaptation à la question posée par le demandeur et à l'usage que celui-ci veut faire de la réponse qui lui est fournie. Cela n'exige pas nécessairement un niveau d'incertitude très faible, toujours coûteux à atteindre.

Il est aussi essentiel de rappeler que la première source d'erreur est l'échantillonnage. Pour être pleinement exploitable, toute analyse devrait être exécutée sur des produits issus d'un échantillonnage réalisé de manière à assurer leur représentativité, avec une incertitude associée. Il semble difficile de réaliser cette étape critique sans implication de l'analyste lui-même [18].

\section{Les limites de la métrologie en chimie}

\section{L'analyse des traces}

Horwitz a montré à partir de l'exploitation de plus de 10000 résultats fournis par des laboratoires de réputation reconnue, utilisant des techniques différentes, sur des échantillons de provenance et de nature très diverses, pour des analyses de composés dont la concentration variait depuis le composé pur jusqu'aux ultratraces, que l'incertitude relative sur l'ensemble des mesures, est une fonction exponentielle de la concentration [2, 38, 44]. Au niveau des traces, l'incertitude augmente fortement et, au-dessous du $\mathrm{mg} / \mathrm{kg}$, il est fréquent qu'elle soit de l'ordre de grandeur de la mesure elle-même. Pourtant les développements actuels de l'instrumentation analytique permettent d'atteindre des limites de quantification suffisantes même au niveau des traces. L'automatisation et les couplages de techniques facilitent le contrôle du processus analytique et des résultats. Certaines erreurs instrumentales et humaines diminuent ainsi fortement, surtout celles qui sont liées au système de mesure. Mais d'autres erreurs qui ont essentiellement pour origine la nature de l'échantillon (son inhomogénéité, les impuretés de la matrice) et les étapes d'extraction et de prétraitement, restent difficilement contrôlables. Selon la technique d'analyse, elles peuvent entraîner des contaminations, des interférences ou des pertes et prennent une importance considérable en analyse de traces. Il y a là une différence fondamentale avec la mesure en physique où l'échantillon n'influe pas sur la mesure ni sur l'incertitude car les manipulations y sont simples; les erreurs sont essentiellement liées à des procédés de mesure directs.

\section{L'analyse de spéciation}

Il s'agit d'analyser, souvent au niveau des traces, les différentes espèces chimiques (organiques, organométalliques, ioniques), dans lesquelles un même élément peut être présent, souvent dans une matrice elle-même complexe (échantillons environnementaux et biologiques). En plus des incertitudes résultant des concentrations faibles, la complexité 
physico-chimique des échantillons entraîne toutes les difficultés que l'on peut rencontrer en analyse chimique :

- il faut réaliser l'extraction, la mise en solution, la séparation, éventuellement la dérivation et l'analyse de chaque espèce chimique contenant l'élément sans la modifier; ces protocoles complexes doivent être adaptés à chaque type d'échantillon suivant le milieu ;

- les différentes espèces à analyser sont en équilibres chimiques plus ou moins stables et mal connus; les étapes de préparation peuvent entraîner des déplacements chimiques qui suppriment la traçabilité ;

- même si des protocoles existent qui permettent d'isoler les différentes formes chimiques des espèces à analyser, il n'existe que rarement des composés de référence exactement adaptés aux échantillons.

Pour tenter de maintenir une traçabilité, on s'efforce d'élaborer des matériaux de référence d'origine naturelle, contenant les substances à analyser dans des milieux types les plus diversifiés. Il s'agit là d'une quête sans fin devant le nombre et la diversité des échantillons naturels. Les matériaux dopés ne sont qu'une solution imparfaite. Des essais interlaboratoires aident à mettre au point des méthodes pour développer de plus en plus de MRC et pour aboutir à la sélection de méthodes de référence convenables.

Une traçabilité rigoureuse n'est donc que rarement envisageable actuellement (Tab. II). En l'absence de méthodes idéales qui permettraient d'accéder à chaque espèce in situ, la confiance que l'on peut accorder à un résultat ne peut que dépendre de l'utilisation qualité des protocoles analytiques les mieux adaptés (campagnes interlaboratoire), de la rigueur de la mise en œuvre de techniques analytiques bien validées et contrôlées, et de la compétence d'analystes travaillant en assurance qualité. En analyse de spéciation, la traçabilité d'un résultat aux unités fondamentales n'est pas établie. La traçabilité que l'on peut envisager peut seulement être établie par rapport à une méthode de référence, ou un MRC lorsqu'il existe.

\section{Conclusion : quelles réponses aux besoins des utilisateurs?}

\section{Métrologie et réalité de l'analyse chimique}

Le schéma conceptuel simple sur lequel a été établie la métrologie en physique ne convient pas face aux difficultés concrètes que rencontrent les chimistes pour déterminer une quantité de substance. La traçabilité aux unités de base ne peut que rarement être réalisée, faute de méthodes primaires en nombre suffisant et adaptées à la nature complexe des échantillons. Les méthodes primaires disponibles ont des domaines et des potentiels d'application beaucoup trop limités face aux besoins. La plupart des analyses doivent donc être réalisées par des méthodes relatives, la traçabilité étant alors assurée au moyen des matériaux de référence certifiés utilisés pour l'étalonnage. Cependant, il n'existe pas toujours de MRC correspondant à l'échantillon et le protocole analytique peut présenter des lacunes de traçabilité.

Dans l'état actuel des techniques analytiques, on est donc contraint d'admettre que la traçabilité en chimie ne peut pas posséder le caractère absolu qu'elle possède en physique. Le point de référence de la chaîne de traçabilité est souvent le MRC utilisé pour l'étalonnage plutôt que la mole ou le kilogramme. Lorsqu'aucun MRC n'est disponible, les résultats sont seulement traçables à la méthode utilisée. Celle-ci constitue alors le point de référence et devient même le point ultime de la traçabilité (Fig. 1). La méthode d'analyse joue alors un rôle métrologique primordial puisque c'est elle qui garantit la comparabilité des résultats, critère fondamental de la qualité de l'analyse.

\section{Les besoins et les développements}

L'activité analytique est portée par la demande économique constante et les contraintes réglementaires. Pour y répondre d'importants efforts sont nécessaires pour développer les outils qui peuvent améliorer la comparabilité et la traçabilité des mesures. Une stratégie intégrée doit être envisagée pour attaquer les problèmes à tous les niveaux :

- en recherche, aussi bien fondamentale que technologique et instrumentale, pour développer de nouvelles méthodes de mesure, spécifiques, susceptibles de constituer des méthodes primaires ou définitives, ou pour améliorer les méthodes existantes, de prétraitement, purification et séparation en particulier ;

- pour élaborer de nouveaux matériaux de référence primaires, certifiés en ce qui concerne leur pureté et leur stœchiométrie, avec une incertitude établie ;

- pour élaborer des MRC à matrice, nécessaires en nombre croissant dans les milieux les plus divers, traçables à des unités ou des substances de références reconnues;

- pour proposer des matériaux de référence de travail, destinés à des tests de contrôle de qualité interne ou externe, permettant aux laboratoires d'évaluer la qualité des méthodes qu'ils mettent en œuvre ;

- pour multiplier les méthodes de référence ;

- des modèles doivent être proposés aux analystes pour établir l'incertitude réelle des mesures, de manière à en évaluer aussi toutes les composantes.

Mais la réponse à un besoin dans un domaine particulier ne peut pas être utilisée dans un autre domaine de la chimie analytique; des approches alternatives doivent être recherchées et mises en œuvre à chaque fois. La tâche de l'analyste est d'adapter les outils dont il dispose pour que la comparabilité et la traçabilité des mesures se situent au plus haut niveau possible en s'efforçant de suivre une chaîne de traçabilité aboutissant à une référence reconnue (matériau ou méthode). L'analyse chimique doit d'abord reposer sur les bases fondamentales des sciences analytiques, source d'un développement méthodologique et instrumental permanent, 
indépendamment de tout aspect de service. Le travail analytique aboutissant à l'identification et à la mesure doit ensuite être réalisé dans le cadre de systèmes «qualité » adéquats, par des méthodes adaptées et validées, garantissant que les résultats fournis correspondent aux objectifs de l'utilisateur.

En conséquence, au niveau structural, il est nécessaire de coordonner les efforts sectoriels (analyses environnementales, agro-alimentaires, contrôle des produits, répression des fraudes, etc.) pour optimiser le choix des outils (substances certifiées et de référence, méthodes de référence) et faciliter leur accès. Ceci passe par une coordination de l'activité entre laboratoires et instituts en charge des mesures dans les différents secteurs d'activité. Ceci nécessite sûrement de stimuler les efforts de certains instituts pour supporter des projets/programmes de recherche en métrologie en chimie. Une collaboration internationale pour faciliter et accélérer ces développements est nécessaire. Enfin, les domaines prioritaires sur lesquels doivent porter les efforts de recherche doivent être sélectionnés en relation avec les acteurs économiques et sociaux.

\section{Références}

1. Vocabulaire international de métrologie, norme NF x 07001, AFNOR, 1994.

2. Boyer, K. W.; Horwitz, W.; Albert, R. Anal. Chem. 1985, 57, 454.

3. King, B. Analyst 1993, 118, 587-591.

4. Leroy, M.; Boos, A.; Maier, E. A.; Griepink, B. Qualité et assurance qualité en chimie analytique, Collection Techniques de l'Ingénieur, Traité analyse et caractérisation. Doc P 280.

5. Rios, A.; Valcarcel, M. Analyst 1994, 119, 109-112.

6. Valcarcel, M.; Rios, A. Analyst 1995, 120, 2291-2297.

7. Thompson, M. Analyst 1996, 121, 285-288.

8. Alexandrov, Y. I. Analyst 1996, 121, 1137-1145.

9. King, B. Analyst 1997, 122, 197-204.

10. Alexandrov, Y. I. Fresenius J. Anal. Chem. 1997, 357, 563571.

11. Kaarls, R.; Quinn, T. J. Metrologia 1997, 34, 1-6.

12. Richter, W.; Dube G. Metrologia 1997, 34, 13-18.

13. Pan, R. X. Metrologia 1997, 34, 35-40.

14. King, B. Metrologia 1997, 34, 41-48.

15. Quinn, T. J. Metrologia 1997, 34, 61-66.

16. De Bievre, P.; Taylor, P. D. P. Metrologia 1997, 34, 67-76.

17. Metrology in Chemistry and Biology: A Practical Approach, SMT 4 - CT 96 - 6505. 1998, SMT Programme, Commission Européenne, Bruxelles.

18. Gy, P. Hétérogénéité, échantillonnage, homogénéisation, Paris : Masson, 1988.
19. BIPM - Comptes rendus de la $14^{\mathrm{e}}$ Conférence des Poids et Mesures, 1971.

20. BIPM - Comité Consultatif pour la Quantité de Matière, 1985, 1.

21. Dube, G.; Henrion, A.; Richter, N. Metrologia 1997, 34, 8386.

22. Watters, R. A.; Eberhardt, K.; Beary, E.; Fassett, J. Metrologia 1997, 34, 87-96.

23. Bowers, G. N.; Fassett, J. P.; White, E. Anal. Chem. 1993, 65 (12), 475R.

24. Ramendik, G. I. Fresenius J. Anal. Chem. 1997, 357, 233-240.

25. ICH, Validation of Analytical Procedures, Genève, 1996.

26. Calmet, D. Analusis, à paraître dans ce dossier.

27. Feinberg, M. La validation des méthodes d'analyse, Paris : Masson, 1996.

28. Skoog, A.; Leary, J. J. Principles of Instrumental Analysis, Saunders College Publishing, Orlando, Florida, 1992.

29. Certification of Reference Materials. General and Statistical Principles ISO-IEC Guide 35 - 1985.

30. Guidelines for the production and certification of BCR reference materials, Doc. BCR/48/93. SMT Programme, Commission Européenne, 1994, Bruxelles.

31. Quevauviller, P. Analusis 1993, 21(2), M 47 - M 53.

32. Quevauviller, P. Fresenius J. Anal. Chem. 1996, 354, 515-520.

33. Quevauviller, P. Analusis 1997, 25, 2, M17-M21.

34. Hasselbarth, W. Fresenius J. Anal. Chem. 1996, 354, 263-265.

35. Directory of Certified Reference Materials, IAEA/R6/128 (Rev 1), Secretary of REMCO, ISO, Genève.

36. Physico-Chemical Measurements. Catalogue of Reference Materials from National Laboratories, Pure Appl. Chem. 1976, $48,503$.

37. Standard and Reference Materials for Marine Science, Technical Memorandum, NQJ OMA 51 NOAA, US Department of Commerce, 6001 Executive Blvd, Rockville MD 20852, 1989.

38. Horwitz, W.; Albert, R. Analyst 1997, 122, 615-617.

39. Guide pour l'expression de l'incertitude des mesures, ISBN 92-67-20188-3 1995, ISO, Genève.

40. Quantifying Uncertainty in Analytical Measurements, EURACHEM Guide, TW11 DNH. 1995, Teddington, Middlesex, $\mathrm{UK}$

41. De Bievre, P.; Peiser, H. S. Metrologia 1997, 34, 49-59.

42. Atomic Weights of the Elements, 1995, Pure Appl. Chem. 1996, 68, 2339-2359

43. Analytical Method Committee, Analyst 1995, 120, 2291-2297.

44. Albert, R.; Horwitz, W. Anal. Chem. 1997, 69, 789-790.

45. Maier, E. A.; Griepink B. in : Kellner et al., Analytical chemistry, Chapter 3: Quality control and quality assurance, $\mathrm{VCH}$ Verlagsgesellschaft mbH: Weinheim, Germany, 1997. 